OPEN ACCESS

Edited by:

Luigi Marano,

University of Siena, Italy

Reviewed by:

Jian Bin Li,

Shandong Cancer Hospital, China

Ludovico Carbone,

University of Siena, Italy

*Correspondence:

Fan Wang

wangfan1965@126.com

Specialty section:

This article was submitted to Gastrointestinal Cancers:

Gastric Esophageal Cancers,

a section of the journal

Frontiers in Oncology

Received: 29 September 2021 Accepted: 20 December 2021

Published: 18 January 2022

Citation:

Gui Z, Liu H, Shi W, Xu Y, Qian H and Wang $F$ (2022) A Nomogram for

Predicting the Risk of Radiotherapy-

Related Esophageal Fistula in Esophageal Cancer Patients.

Front. Oncol. 11:785850.

doi: 10.3389/fonc.2021.785850

\section{A Nomogram for Predicting the Risk of Radiotherapy-Related Esophageal Fistula in Esophageal Cancer Patients}

\author{
Zhongxuan Gui, Huiquan Liu, Weijiong Shi, Yuechen Xu, Han Qian and Fan Wang* \\ Department of Radiation Oncology, The First Affiliated Hospital of Anhui Medical University, Hefei, China
}

Background: To construct and validate a nomogram for predicting the risk of esophageal fistula in esophageal cancer patients receiving radiotherapy.

Methods: A retrospective nested case-control study was performed, in which a total of 81 esophageal fistula patients and 243 controls from 2014 to 2020 in the First Affiliated Hospital of Anhui Medical University were enrolled. Factors included in the nomogram were determined by univariate and multiple logistic regression analysis. The following methods including ROC curve, C-index, calibration curves, Brier score, and decision curve analysis (DCA) were adopted to evaluate this nomogram.

Results: Multivariate logistic regression analysis showed that T4 stage, level 4 stenosis, ulcerative esophageal cancer, prealbumin, and maximum diameters of GTV and NLR were the independent risk factors of esophageal fistula. Accordingly, a nomogram incorporating the aforementioned six parameters was constructed. The AUC was 0.848 (95\% $\mathrm{Cl} 0.901-0.895)$, indicating a high prediction accuracy of this nomogram. Further evaluation of this model showed that the C-index was 0.847 , while the bias-corrected Cindex after internal validation was 0.833 . The Brier score was 0.127 . The calibration curves presented good concordance, and the DCA revealed promising clinical application.

Conclusions: The nomogram presents accurate and applicable prediction for the esophageal fistula risk in esophageal cancer patients receiving radiotherapy.

Keywords: esophageal cancer, radiotherapy, esophageal fistula, risk factors, nomogram

\section{INTRODUCTION}

Esophageal cancer (EC) is the seventh most common malignancy worldwide leading to estimated 544,000 deaths in 2020 (1). Patients with EC are usually diagnosed at the advanced or metastatic stage due to the lack of early symptoms and the rapid progression of carcinoma. Thus, a considerable proportion of EC patients are considered inoperable or surgically contraindicated at the initial visit. Radiotherapy, especially the intensity-modulated radiation therapy (IMRT), plays a critical role in the treatment of locally advanced inoperable EC (2). It is remarkable that esophageal fistula $(\mathrm{EF})$, a fatal treatment-related complication, may occur during and after radiotherapy. 
The incidence of EF in EC patients receiving chemoradiotherapy is about $4.3 \%-22 \%$ according to previous studies (3-10). The common clinical symptoms of EF include bucking, back/chest/ abdominal pain, fever, hydrothorax, dysphagia, and empyema (11). Therefore, early prediction of EF and appropriate intervention are important to enhance clinical outcomes and increase quality of life.

Previous literature $(6,8,12)$ described that several clinical parameters are closely correlated with the occurrence of EF, including age, $\mathrm{T}$ stage, $\mathrm{N}$ stage, stenosis, ulceration, low serum cholesterol level, and body mass index (BMI). However, a unified diagnosis criterion for esophageal stenosis has not been unified, and the majority of studies defined stenosis solely based on symptoms $(4,8,13)$. To date, there are still no reliable clinical standards for predicting high-risk EF. In this study, we further refined several EF-associated parameters and explored a clinically applicable nomogram to predict EF risk for EC patients receiving radiotherapy.

\section{MATERIALS AND METHODS}

\section{Study Design}

We retrospectively studied the medical records of EC patients receiving radiotherapy in the First Affiliated Hospital of Anhui Medical University, between October 19, 2014, and June 15, 2020. Follow-up was carried out since the radiotherapy stated until the EF occurred or until June 15, 2021, ensuring that each patient was followed for sufficient time to accurately assess the occurrence of EF. The enrolled EC patients with previous malignancies, history of esophageal surgery, already formed fistula before treatment, and lost follow-up were excluded. The inclusion criteria for EF patients are applied: (1) histologically proved squamous cell carcinoma, adenocarcinoma, or small cell carcinoma of the esophagus; (2) complete record of the necessary clinical characteristics; (3) clinically confirmed EF or esophageal perforation which were detected by endoscopy, computed tomography (CT), or esophagography; and (4) no EF before radiotherapy. The diagnostic standards of EF were as follows: (i) iodine examination shows that contrast media leak out from the patient's fistula, or into the patient's chest, mediastinum; (ii) CT scan findings include mediastinal air surrounding the esophagus, abscess cavities adjacent to the esophagus in the pleural space, mediastinal air, pleural effusion, pneumothorax, and subdiaphragmatic air(11). To improve the comparison and the stability of the results, the cases and controls were matched by age, gender, and diagnosis time at a ratio of $1: 3$. This retrospective nested case-control study was approved by the institutional research ethics committee of Anhui Medical University.

\section{Data Collection}

In this single-centered, retrospective study, we obtained the demographic characteristics, laboratory data, radiological examinations, and therapeutic strategy from electronic medical records. The following clinical characteristics were collected before radiotherapy: general characteristics (gender, age, body mass index (BMI), smoking history, hypertension, diabetes $(\mathrm{DM})$ ), tumor characteristics (stage, location, ulcerative EC, esophageal stenosis), treatment characteristics (re-radiotherapy, radiotherapy dose, chemotherapy, gross tumor volume (GTV), maximum diameter of GTV, length of GTV, treatment response), and hematological data (albumin, hemoglobin, prealbumin, neutrophil count, lymphocyte count).

The pretreatment clinical staging was on the basis of the American Joint Committee on Cancer (AJCC) 8th edition staging system (14). GTV was defined by the planning physicians as the primary tumor (GTVp) and involved mediastinal and hilar nodes (GTVn) found by computed tomography (CT) or positron emission tomography/computed tomography (PET/CT) before treatment. The NLR was defined as the absolute neutrophil count divided by the absolute lymphocyte count. The treatment response was assessed 30 days after radiotherapy by enhanced CT based on Response Evaluation Criteria in Solid Tumors (RECIST) version 1.1, and it was classified as clinically complete response (CR), partial response (PR), stable disease (SD), or progressive disease (PD). $\mathrm{CR}$ was defined as the disappearance of all target lesions, $\mathrm{PR}$ as reduction by $30 \%$ or more in maximum diameter of target lesion, PD as increase by $20 \%$ or more in the longest tumor diameter of target lesion or appearance of new lesions, and SD as other than $\mathrm{CR}, \mathrm{PD}$, and PR.

To determine the stenosis of esophagus, we reviewed the esophagography image obtained before radiotherapy and measured the lumen diameter at the widest part of the oral side (Figure 1A) and the narrowest part of the lesion (Figure 1B). The stenosis ratio was calculated as following formula: $c=(a-b) / a * 100 \%$. The severity of esophageal stenosis (stenosis ratio) was evaluated and classified as the following grades: grade I, $0 \%-24 \%$; grade II, $25 \%-49 \%$; grade III, $50 \%-74 \%$; grade IV, $75 \%-100 \%$.

\section{Statistical Analyses}

Odds ratios (OR) with 95\% confidence intervals (CI) were used to evaluate the relationship between the clinical parameters and $\mathrm{EF}$, and the best cutoff values to predict EF risk were determined using receiver operating characteristic (ROC) curves. We investigated all the clinical factors by univariable logistic regression for paired samples, and the significant factors were included in the multivariable logistic-regression model. Statistical analyses were performed using IBM SPSS 22.0 software. A nomogram integrating independent risk factors of $\mathrm{EF}$ was created using $\mathrm{R}$ software (version 3.6.1). The receiver operating characteristic (ROC) curve and the area under the ROC curve (AUC) were applied to assess the discrimination of the model. We adopted three methods, including C-index for discrimination, calibration curves, and Brier score to evaluate this nomogram. The established nomogram was further internally validated by bootstrapping ( 1,000 bootstrap replicates) to obtain bias-corrected predictive parameters. Significance was defined as 2 -sided $p$-value of $<0.05$. 


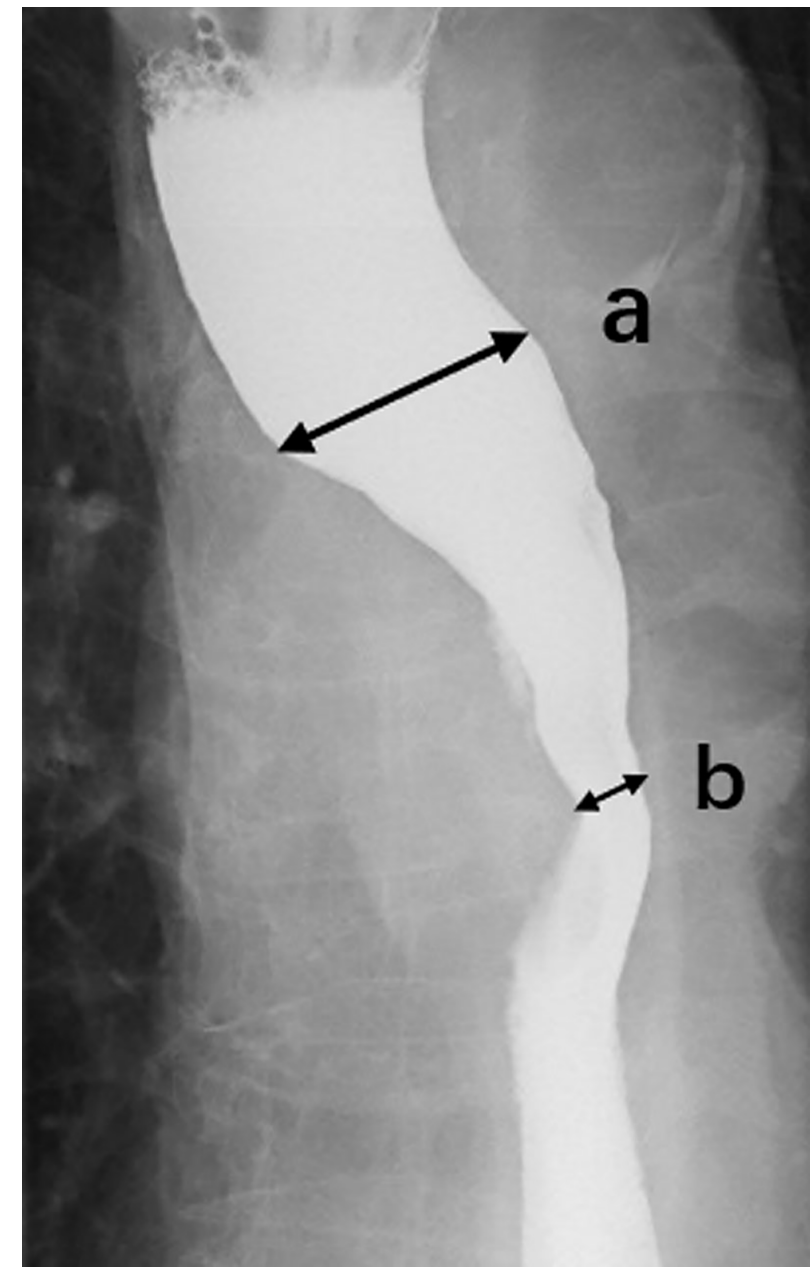

FIGURE 1 | Esophagography image. We reviewed the esophagography image obtained before radiotherapy and measured the lumen diameter at the widest part $(\mathbf{A})$ of the oral side and the narrowest part $(\mathbf{B})$ of the lesion, then calculated the stenotic ratio $(c=(a-b) / a * 100)$.

\section{RESULTS}

\section{Characteristics of Participants}

Between October 19, 2014, and June 15, 2020, 1,894 cases who had undergone radiotherapy were identified in our database and 711 cases were excluded based on our exclusion criteria. Among the 1,183 EC patients, 81 (6.85\%) had developed EF before June 15, 2021. After matching by age, gender, and diagnosis time, a total of 324 EC patients, including those 81 (25.0\%) EF cases and $243(75.0 \%)$ controls, were enrolled for the subsequent analyses. The follow-up period ranged from 2.3 to 82.6 months, and the median time was 51.7 months. The median age of these participants was 70.0 years, and the male-to-female ratio is 3.3:1. Middle thoracic (40.7\%) EC was more common than upper thoracic (31.1\%) and lower thoracic (28.0\%) EC. Of these patients who developed EF, 12 patients suffered perforation during RT, while 69 patients developed this complication after RT. The median intervals between the end of radiotherapy and the EF onset were 4.60 months (95\% CI: 3.50-5.64). Among all the 81 cases with EF, 46 cases developed esophagomediastinal fistula, 28 cases developed esophagotracheal fistula, 2 cases developed esophagoarterio fistulas, and 5 cases suffered both esophagomediastinal and esophagotracheal fistula. Managements of fistula included nutrient canal in 61 patients (75.3\%), esophageal stent in 16 patients $(19.7 \%)$, and parenteral nutrition in 4 patients $(4.9 \%)$. The patient characteristics are listed in Table $\mathbf{1}$.

\section{Risk Factors for EF}

As shown in Table 2, univariate analysis revealed that BMI $<20$ $\mathrm{kg} / \mathrm{m}^{2}, \mathrm{~N} 1-3$ stage, T4 stage, NLR, hemoglobin, prealbumin, reradiotherapy, ulcerative EC, stenosis, length of GTV, and maximum diameter of GTV were significantly correlated with the occurrence of EF ( $p$-value $<0.05)$. The other clinical parameters including age, albumin, tumor location, $M$ stage, total dose > $60 \mathrm{~Gy}$, single dose, GTV volume, chemotherapy, treatment response $(\mathrm{PR}+\mathrm{CR}$ vs. $\mathrm{SD}+\mathrm{PD})$, smoking history, diabetes, and hypertension were not significant for their association with EF. Multivariate analysis showed that T4 stage, level 4 stenosis, ulcerative EC, prealbumin, and maximum diameters of GTV and NLR remained significant ( $p$-value < $0.05)$, which indicated that these clinical characteristics were independent risk factors for the occurrence of EF (Table 2).

\section{Predictive Nomogram for EF}

According to the results of multivariate analysis, a nomogram incorporating the 6 independent risk factors was constructed to predict EF (Figure 2). The total point was calculated with the use of T4, NLR, ulcerative EC, level 4 stenosis, prealbumin, and maximum diameter of GTV. The point of each of these variables was given a score on the point scale axis. A total score could be easily calculated by adding each single score, and by projecting the total score to the lower total point scale, we were able to estimate the probability of EF.

\section{Evaluation and Validation the Nomogram}

The AUC was 0.848 (95\% CI 0.901-0.895) (Figure 3A), indicating robust discrimination. The Brier score of the nomogram was 0.127 , which was close to 0 , indicating great predictive ability (Figure 3B). As shown in Figure 3C, the calibration plot showed good conformity between predicted and actual probability for EF. The uncorrected concordance index (C-index) was 0.847 , and the corrected $\mathrm{C}$-index generated by internal validation was 0.833 (Figure 3C). Finally, we performed a decision curve analysis (DCA) to evaluate the clinical utility of the nomogram and its effective threshold ranged from approximately $7 \%$ to $91 \%$, showing that using this nomogram was more effective than the "treat-all" or the "treatnone" strategy in predicting EF when the prediction probability was within this range (Figure 3D).

\section{DISCUSSION}

The EF, a severe complication, deteriorates the quality of life and shortens survival in EC patients. Predicting EF risk is crucial for developing individual therapeutic strategies. In this study, we 
TABLE 1 | The characteristics of patients with radiotherapy-related esophageal fistula.

\section{Characteristics}

\section{Gender}

Male

Female

Age (years)

$<60$

$\geq 60$

History of smoking

No

Yes

History of hypertension

No

Yes

History of diabetes

No

Yes

BMl $\left(\mathrm{kg} / \mathrm{m}^{2}\right)$

$<20$

$\geq 20$

T stage

T1-3

$\mathrm{T} 4$

$\mathrm{N}$ stage

NO

N1-3

$\mathrm{M}$ stage

MO

M1

Location of primary tumor

Upper thoracic esophagus

Middle thoracic esophagus

Lower thoracic esophagus

Ulcerative tumor

No

Yes

Maximum diameter of GTV $(\mathrm{cm})$

$\leq 2.5$

$>2.5$

Length of GTV (cm)

$\leq 5.5$

$>5.5$

GTV volume $\left(\mathrm{cm}^{3}\right)$

$\leq 60$

$>60$

Fraciton dose (Gy)

1.8

2.0

Total radiation dose

$<60$

$\geq 60$

Re-radiotherapy

No

Yes

Treatment modalities

Concurrent CRT

Sequential CRT

Without CT

Treatment response

$\mathrm{SD}+\mathrm{PD}$

$\mathrm{CR}+\mathrm{PR}$

Stenosis before radiotherapy

Levels 1-3

Level 4
No esophageal fistula

Esophageal fistula

$186(0.77)$

$57(0.23)$

$62(0.77)$

$19(0.23)$

$41(0.17)$

$20(0.25)$

$202(0.83)$

$61(0.75)$

$168(0.69)$

$75(0.31)$

$48(0.59)$

$33(0.41)$

$212(0.87)$

$31(0.13)$

$67(0.83)$

$14(0.17)$

$235(0.97)$

$8(0.03)$

$76(0.94)$

$5(0.06)$

$93(0.38)$

$150(0.62)$

$43(0.53)$

$38(0.47)$

$224(0.92)$

19 (0.08)

55 (0.68)

26 (0.32)

$104(0.43)$

$139(0.57)$

$25(0.31)$

$56(0.69)$

189 (0.78)

$54(0.22)$

$66(0.81)$

$15(0.19)$

$74(0.30)$

95 (0.39)

74 (0.30)

$191(0.79)$

$52(0.21)$

$72(0.30)$

$171(0.70)$

$141(0.58)$

$102(0.42)$

$151(0.62)$

92 (0.38)

$17(0.07)$

226 (0.93)

$148(0.58)$

$92(0.42)$

$231(0.95)$

12 (0.05)

127 (0.52)

$103(0.42)$

$13(0.05)$

27 (0.33)

$37(0.46)$

$17(0.21)$

$34(0.42)$

$47(0.58)$

7 (0.09)

74 (0.91)

$28(0.35)$

$53(0.65)$

$36(0.44)$

45 (0.56)

$10(0.12)$

$71(0.88)$

$36(0.54)$

$45(0.46)$

$70(0.86)$

$11(0.14)$

$38(0,47)$

$39(0.48)$

4 (0.05)

$106(0.44)$

$31(0.38)$

50 (0.62)

$18(0.22)$

$63(0.78)$ 
TABLE 1 | Continued

\begin{tabular}{l} 
Characteristics \\
\hline Hemoglobin (g/L) \\
$<120$ \\
$\geq 120$ \\
Albumin (g/L) \\
$\quad<35$ \\
$\geq 35$ \\
Prealbumin (mg/L) \\
$<180$ \\
$\geq 180$ \\
NLR \\
$<3.2$ \\
$\geq 3.2$ \\
PLR \\
$<155$ \\
$\geq 155$
\end{tabular}

MI, body mass index; GTV, gross tumor volume; CRT, chemoradiotherapy; CT, chemotherapy; SD, stable disease; PD, progressive disease; CR, complete response; PR, partial response; NLR, neutrophil to lymphocyte ratio; PLR, platelet to lymphocyte ratio.

comprehensively evaluated the fistula-related parameters and identified several new independent risk factors. For dosimetryrelated indicators, we found that the incidence of fistula was $28.9 \%$ in the group of GTV maximum diameters $\leq 2.5 \mathrm{~cm}$, while the incidence decreased to $14.6 \%$ in the group of maximum diameter of GTV $>2.5 \mathrm{~cm}$. To some extent, the maximum diameter of GTV indicated the severity of local radiation damage and the depth of tumor invasion. These results suggest that for the high-risk EF patients, moderate shrinks of GTV are needed.

The status when esophageal carcinoma invades adjacent structures, such as the pleura, pericardium, azygos vein, diaphragm, peritoneum, aorta, vertebral body, and airway, is defined as T4 stage in the 8th Edition of the AJCC TNM Staging System (15). Recently, Chen et al. (16)revealed that the incidence rate of EF was $30.1 \%$ in EC patients (stage T4b) and the median overall survival was only 6.9 months. It is easy to understand that if the space-occupying lesions were eliminated speedily by chemoradiotherapy without sufficient tissue repair, the fistula might form between the esophageal lumen and contiguous structures. Formation of a fistula between the esophagus and the mediastinum was suspected.

We believe that EC with external esophageal invasion should receive individualized radiotherapy not only to kill the tumor cells but also to maximize normal tissue repair. Additionally, we also characterized ulcerative lesion as an independent factor of EF, which was consistent with previous studies $(8,16)$. In our study, we observed that the incidence rate of ulcerative-type carcinoma diagnosed before radiotherapy in the EF group was nearly three times that in the control group (58.0\% vs. $21.3 \%$ ). Ulcerative esophageal cancer has a deep invasion and thin wall, reaching or penetrating the muscular layer, and then the perforation may occur due to increased luminal pressure during swallowing or severe coughing.

It is estimated that more than half of EC patients suffer from malnutrition (17). Cancer-associated dysphagia and anorexia are the leading causes of malnutrition, while radiation-induced mucositis makes matters worse. Malnutrition and cachexia restrain the damage repair, reduce therapeutic effects, and increase mortality $(17,18)$. In this study, we also explored the risk factors from the perspective of nutrition and found that BMI, hemoglobin, and prealbumin were significantly associated with the occurrence of EF. Meanwhile, multivariable analysis demonstrated that low prealbumin was an independent risk factor, which was not previously reported. Previous studies have shown that prealbumin is considered to be more sensitive than albumin in the nutritional assessment of patients undergoing radiotherapy (19). Serum prealbumin with a halflife of 2 to 3 days in the human body is a good clinical marker of protein balance and nutritional status $(20,21)$. These results indicate that nutritional support, such as oral nutritional supplements, promisingly prevents the occurrence of EF.

Most of the published articles investigated esophageal stenosis based on symptoms and did not define its degree of severity. The NCCN guidelines noted that the most common cause of dysphagia is obstruction, but it may also be associated with cancer-related dysmotility (22), which may affect the assessment of esophageal stenosis. Thus, we used a specific criterion to evaluate and grade the stenosis by esophageal barium meal examination before treatment. Intriguingly, our results showed that esophageal stenosis at level 4 was a significant independent risk factor in fistula formation. It is speculated that the internal pressure was associated with severity of the esophageal stenosis and caused expansionary damage to esophageal wall. As a result, it is appropriate to identify the esophageal stenosis before radiotherapy, so as to make dietary adjustments and palliative management, such as endoscopic stenting and endoscopic dilation $(23,24)$.

Malignant tumors usually trigger an intrinsic inflammatory response to establish a tumorigenic microenvironment $(25,26)$. The NLR as a marker of systemic inflammatory response has received great attention because of its accessibility. In clinical practice, the NLR is increasingly used to predict bacteremia, peptic ulcer perforation, severe cholecystitis, acute cholecystitis, acute pancreatitis, acute coronary syndrome and communityacquired infections, and even the survival of cancer patients (2730). Systemic inflammatory responses have been proved to 
TABLE 2 | Univariate and multivariate analysis of the factors associated with esophageal fistula.

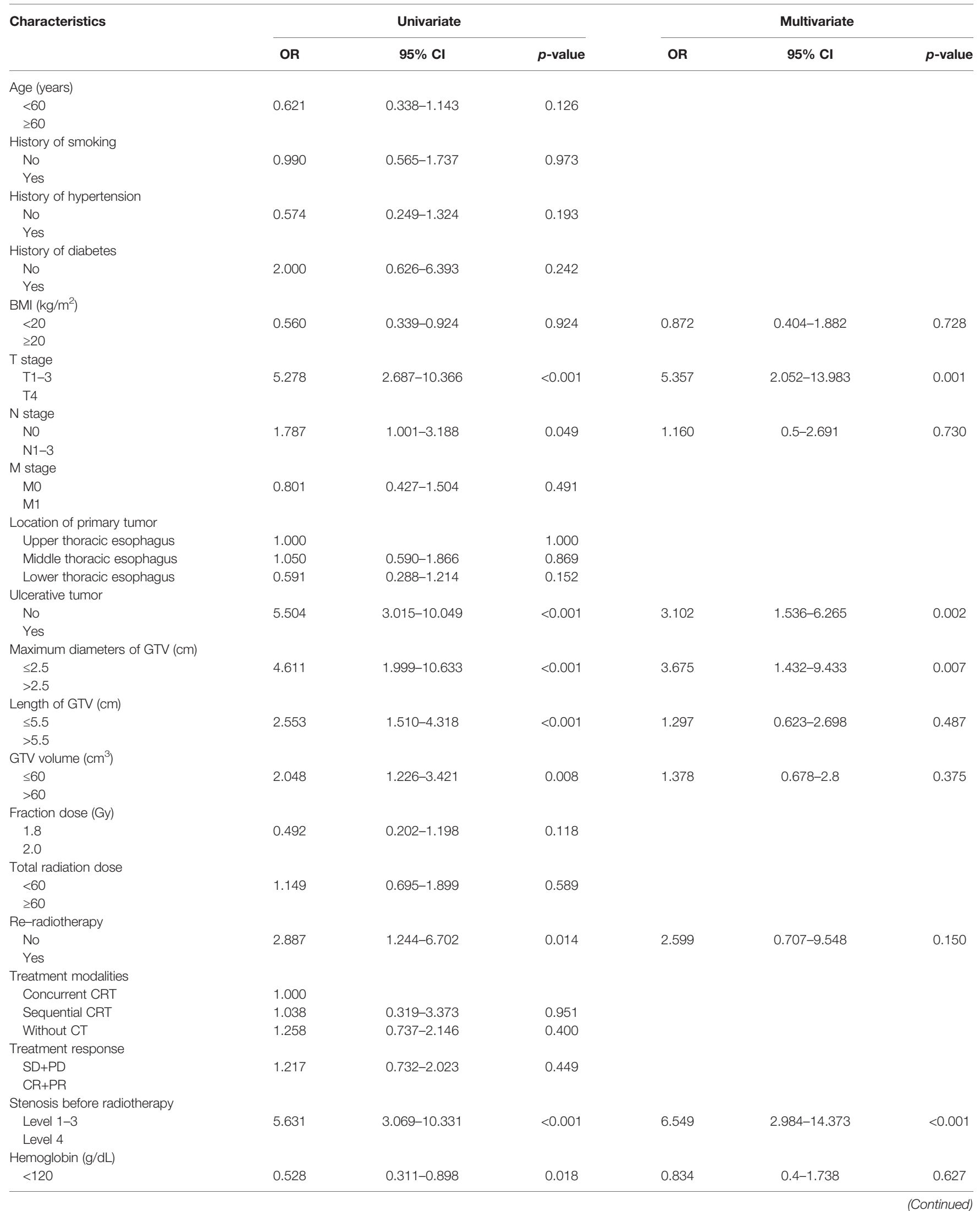


TABLE 2 | Continued

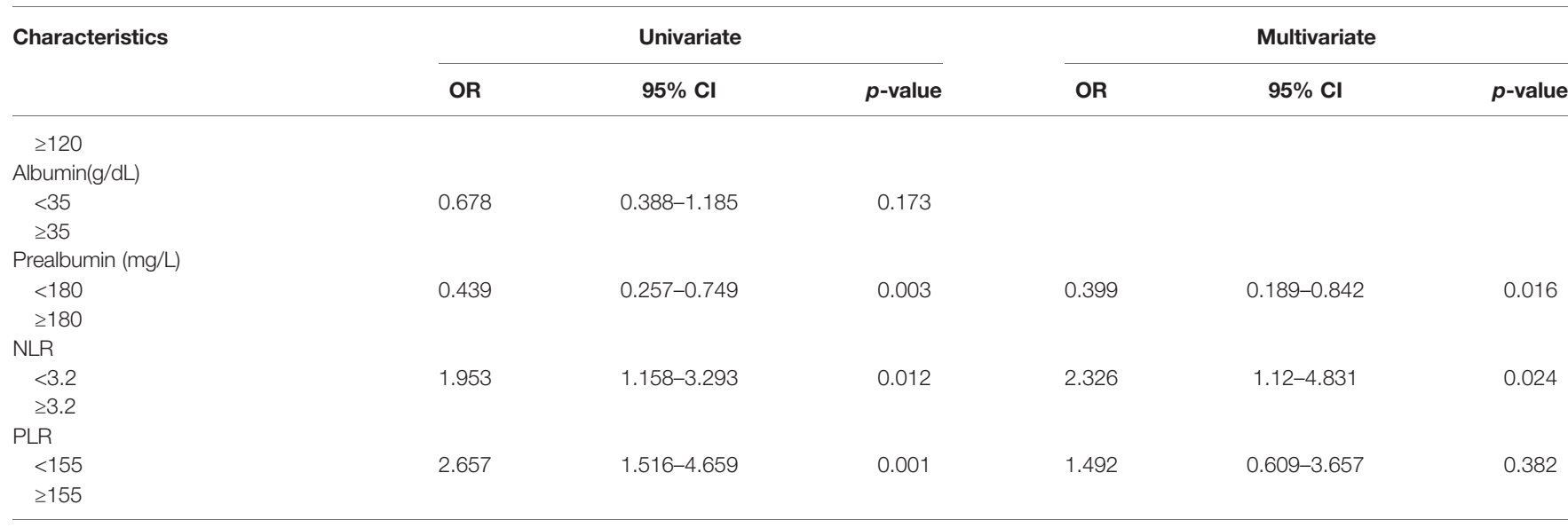

$B M I$, body mass index; GTV, gross tumor volume; CRT, chemoradiotherapy; CT, chemotherapy; SD, stable disease; PD, progressive disease; CR, complete response; PR, partial response; NLR, neutrophil to lymphocyte ratio; PLR, platelet to lymphocyte ratio.

influence the motility, invasiveness, and survival of malignant cells through upregulating cytokines, such as IL-1 $\beta$, IL-6, IL-7, IL- 8 , and IL-12. The host-cellular response to IL- 8 released by cancer cells enhances neutrophil infiltration, which promotes remodeling of the extracellular matrix and tumor progression (31). High NLR represents more severe inflammation and more advanced disease with aggressive clinical characteristics. In this study, we preliminarily explored the significant association of high NLR with EF. Further research is needed to explore the specific mechanism and the application of NLR in EF.

The nomogram is a kind of visual graph based on the multiple regression model. It integrates several parameters and consists of different length line segments. In this study, we screened out six independent risk factors by multiple regression to establish a personalized prediction model. Further validation proved that this nomogram has good predictive accuracy and clinical application potential. This is the first and comprehensive calculable tool consisting of systemic inflammatory status, nutritional status, and radiation-related parameters to predict EF risk. However, our current study has certain drawbacks that merit discussion. First, as a retrospective, single-center study, it was inevitable to have potential bias. Second, only internal validation was carried out due to limited EF cases. External validation from other centers is necessary to confirm the clinical value of this nomogram. Lastly, the interaction between inflammation and fistula remains obscure, and more trials are needed to clarify the underlying mechanisms. In view of these limitations, we are now planning to expand the sample size of EF patients, further explore predictors with clinical practicability, and improve the model on the basis of the current findings to optimize the prediction of EF.

In summary, we characterized several new clinical parameters as the independent risk factors of EF. A nomogram was accordingly constructed and visualized to facilitate the prediction of EF risk. This calculable tool is promisingly applied in clinical practice to participate in determining individual therapeutic strategies for EC patients.

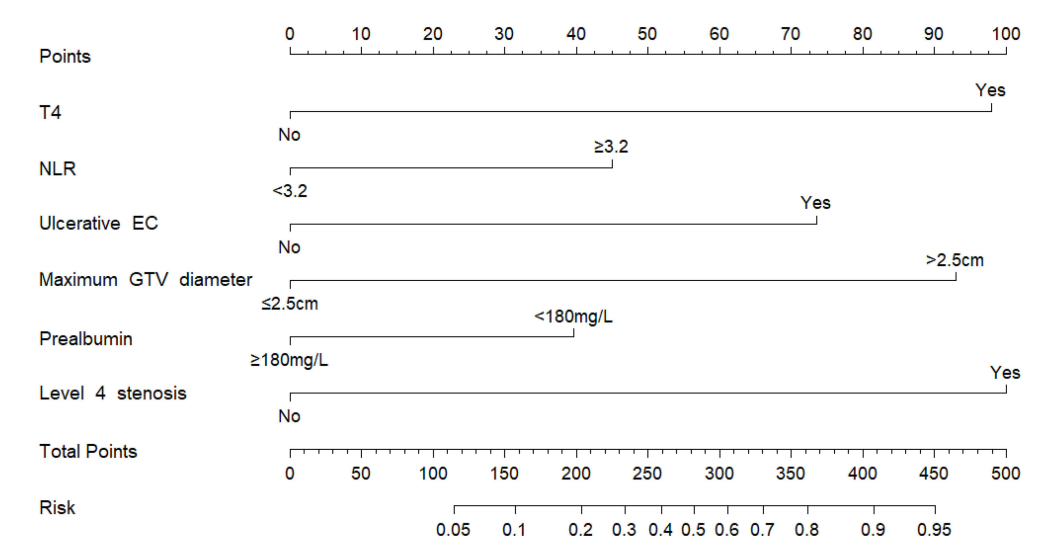

FIGURE 2 | Nomogram for the individualized prediction of radiation-related esophageal fistula in esophageal cancer patients. The nomogram was developed in the cohort, using T4, level 4 stenosis, ulcerative EC, prealbumin, and maximum diameters of GTV and NLR. GTV, gross tumor volume; NLR, neutrophil-to-lymphocyte ratio. 
A

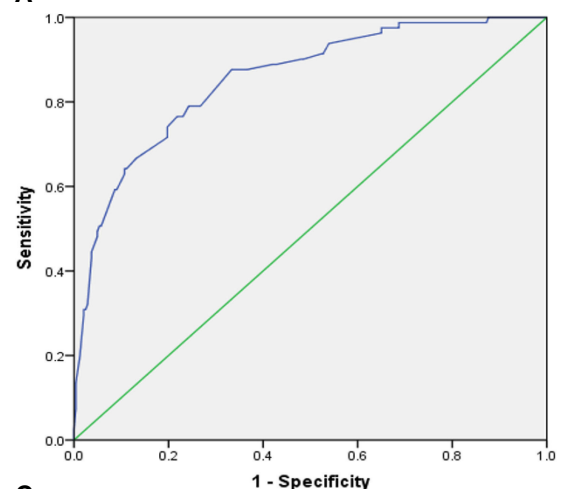

C

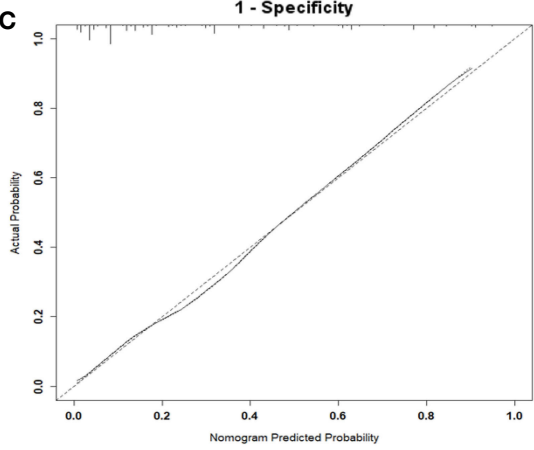

B

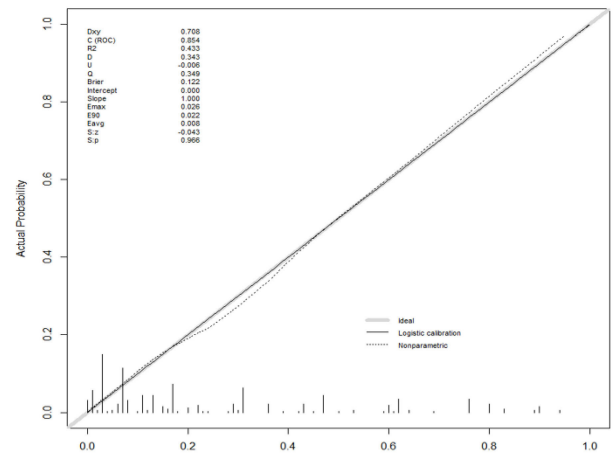

D

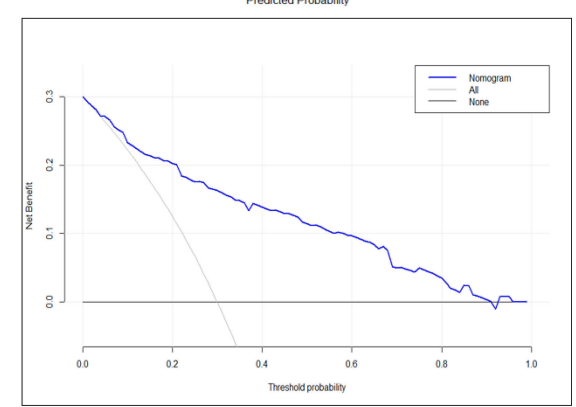

FIGURE 3 | (A) ROC curve for the prediction nomogram. (B) The Brier score for the prediction nomogram. (C) Calibration curve showing nomogram-predicted EF probabilities compared with the actual EF. (D) The decision curve analysis of the nomogram.

\section{DATA AVAILABILITY STATEMENT}

The original contributions presented in the study are included in the article/supplementary material. Further inquiries can be directed to the corresponding author.

\section{ETHICS STATEMENT}

The studies involving human participants were reviewed and approved by the Institutional Review Board of the First Affiliated Hospital of Anhui Medical University. Written informed consent for participation was not required for this study in accordance with the national legislation and the institutional requirements.

\section{REFERENCES}

1. Sung H, Ferlay J, Siegel RL. Global Cancer Statistics 2020: GLOBOCAN Estimates of Incidence and Mortality Worldwide for 36 Cancers in 185 Countries. CA Cancer J Clin (2021) 71(3):209-49. doi: 10.3322/caac.21660

2. Wang SX, Marshall MB. Chemoradiation Therapy as Definitive Treatment of Esophageal Cancer. Surg Clin North Am (2021) 101(3):443-51. doi: 10.1016/ j.suc.2021.03.006

3. Chen Y, Lu Y, Wang Y, Yang H, Xia Y, Chen M, et al. Comparison of Salvage Chemoradiation Versus Salvage Surgery for Recurrent Esophageal Squamous Cell Carcinoma After Definitive Radiochemotherapy or Radiotherapy Alone. Dis Esophagus (2014) 27(2):134-40. doi: 10.1111/j.1442-2050.2012.01440.x

\section{AUTHOR CONTRIBUTIONS}

ZG and HL conceived and designed this study. WS, YX, and HQ processed the data analysis. ZG wrote the article. FW revised the final manuscript. All authors contributed to the article and approved the submitted version.

\section{ACKNOWLEDGMENTS}

The authors appreciate the esophagography images provided by the Department of Medical Imaging at the First Affiliated Hospital of Anhui Medical University.

4. Han D, Zhang J, Zhao J, Lei T, Chen X, Zhang T, et al. Platelet-ToLymphocyte Ratio Is an Independent Predictor of ChemoradiotherapyRelated Esophageal Fistula in Esophageal Cancer Patients. Ann Transl Med (2020) 8(18):1163. doi: 10.21037/atm-20-4053

5. Hihara J, Hamai Y, Emi M, Murakami Y, Kenjo M, Nagata Y, et al. Role of Definitive Chemoradiotherapy Using Docetaxel and 5-Fluorouracil in Patients With Unresectable Locally Advanced Esophageal Squamous Cell Carcinoma: A Phase II Study. Dis Esophagus (2016) 29(8):1115-20. doi: $10.1111 /$ dote. 12433

6. Pao TH, Chen YY, Chang WL, Chang JS, Chiang NJ, Lin CY, et al. Esophageal Fistula After Definitive Concurrent Chemotherapy and Intensity Modulated Radiotherapy for Esophageal Squamous Cell Carcinoma. PLOS ONE (2021) 16(5):e0251811. doi: 10.1371/journal.pone.0251811 
7. Sun X, Han S, Gu F, Lin G, Wang Z, Wang Y, et al. A Retrospective Comparison of Taxane and Fluorouracil-Based Chemoradiotherapy in Patients With Inoperable Esophageal Squamous Cell Carcinoma. J Cancer (2016) 7(9):1066-73. doi: 10.7150/jca.13547

8. Tsushima T, Mizusawa J, Sudo K, Honma Y, Kato K, Igaki H, et al. Risk Factors for Esophageal Fistula Associated With Chemoradiotherapy for Locally Advanced Unresectable Esophageal Cancer: A Supplementary Analysis of JCOG0303. Med (Baltimore) (2016) 95(20):e3699. doi: 10.1097/ md.0000000000003699

9. Watanabe S, Ogino I, Kunisaki C, Hata M. Relationship Between Nutritional Status and Esophageal Fistula Formation After Radiotherapy for Esophageal Cancer. Cancer Radiother (2019) 23(3):222-7. doi: 10.1016/j.canrad. 2018.10.005

10. Zhou ZG, Zhen CJ, Bai WW, Zhang P, Qiao XY, Liang JL, et al. Salvage Radiotherapy in Patients With Local Recurrent Esophageal Cancer After Radical Radiochemotherapy. Radiat Oncol (2015) 10:54. doi: 10.1186/s13014015-0358-z

11. Carrascosa MF, Herreras-Martínez R, Trugeda-Carrera S, Terán Á., Fernández-Díaz MJ, Concha ST, et al. Oesophageal Perforation. Lancet Gastroenterol Hepatol (2018) 3(9):654. doi: 10.1016/s2468-1253(18)30179-1

12. Xu Y, Wang L. Development and Validation of a Risk Prediction Model for Radiotherapy-Related Esophageal Fistula in Esophageal Cancer. Radiat Oncol (2019) 14: (1):181. doi: 10.1186/s13014-019-1385-y

13. Zhang Y, Li Z, Zhang W, Chen W, Song Y. Risk Factors for Esophageal Fistula in Patients With Locally Advanced Esophageal Carcinoma Receiving Chemoradiotherapy. Onco Targets Ther (2018) 11:2311-7. doi: 10.2147/ ott.s161803

14. Rice TW, Patil DT, Blackstone EH. 8th Edition AJCC/UICC Staging of Cancers of the Esophagus and Esophagogastric Junction: Application to Clinical Practice. Ann Cardiothorac Surg (2017) 6(2):119-30. doi: 10.21037/ acs.2017.03.14

15. Daiko H, Kato K. Updates in the 8th Edition of the TNM Staging System for Esophagus and Esophagogastric Junction Cancer. Jpn J Clin Oncol (2020) 50 (8):847-51. doi: 10.1093/jjco/hyaa082

16. Chen B, Deng M, Yang C, Dragomir MP, Zhao L, Bai K, et al. High Incidence of Esophageal Fistula on Patients With Clinical T4b Esophageal Squamous Cell Carcinoma Who Received Chemoradiotherapy: A Retrospective Analysis. Radiother Oncol (2021) 158:191-9. doi: 10.1016/ j.radonc.2021.02.031

17. Mariette C, De Botton ML, Piessen G. Surgery in Esophageal and Gastric Cancer Patients: What is the Role for Nutrition Support in Your Daily Practice? Ann Surg Oncol (2012) 19(7):2128-34. doi: 10.1245/s10434-0122225-6

18. Jordan T, Mastnak DM, Palamar N, Kozjek NR. Nutritional Therapy for Patients With Esophageal Cancer. Nutr Cancer (2018) 70(1):23-9. doi: 10.1080/01635581.2017.1374417

19. Unal D, Orhan O, Eroglu C, Kaplan B. Prealbumin is a More Sensitive Marker Than Albumin to Assess the Nutritional Status in Patients Undergoing Radiotherapy for Head and Neck Cancer. Contemp Oncol (Pozn) (2013) 17 (3):276-80. doi: 10.5114/wo.2013.35281

20. Geisler JP, Linnemeier GC, Thomas AJ, Manahan KJ. Nutritional Assessment Using Prealbumin as an Objective Criterion to Determine Whom Should Not Undergo Primary Radical Cytoreductive Surgery for Ovarian Cancer. Gynecol Oncol (2007) 106(1):128-31. doi: 10.1016/j.ygyno.2007.03.008
21. Guerra LT, Rosa AR, Romani RF, Gurski RR, Schirmer CC, Kruel CD. Serum Transferrin and Serum Prealbumin as Markers of Response to Nutritional Support in Patients With Esophageal Cancer. Nutr Hosp (2009) 24(2):241-2.

22. Ajani JA, Barthel JS, Bentrem DJ, D'Amico TA, Das P, Denlinger CS, et al. Esophageal and Esophagogastric Junction Cancers. J Natl Compr Canc Netw (2011) 9(8):830-87. doi: 10.6004/jnccn.2011.0072

23. Halpern AL, McCarter MD. Palliative Management of Gastric and Esophageal Cancer. Surg Clin North Am (2019) 99(3):555-69. doi: 10.1016/j.suc. 2019.02.007

24. Kim JY, Kim SG, Lim JH, Im JP, Kim JS, Jung HC. Clinical Outcomes of Esophageal Stents in Patients With Malignant Esophageal Obstruction According to Palliative Additional Treatment. J Dig Dis (2015) 16(10):57584. doi: $10.1111 / 1751-2980.12280$

25. Gonzalez H, Hagerling C, Werb Z. Roles of the Immune System in Cancer: From Tumor Initiation to Metastatic Progression. Genes Dev (2018) 32: (1920):1267-84. doi: 10.1101/gad.314617.118

26. Greten FR, Grivennikov SI. Inflammation and Cancer: Triggers, Mechanisms, and Consequences. Immunity (2019) 51(1):27-41. doi: 10.1016/j.immuni. 2019.06.025

27. Han F, Liu Y, Cheng S, Sun Z, Sheng C, Sun X, et al. Diagnosis and Survival Values of Neutrophil-Lymphocyte Ratio (NLR) and Red Blood Cell Distribution Width (RDW) in Esophageal Cancer. Clin Chim Acta (2019) 488:150-8. doi: 10.1016/j.cca.2018.10.042

28. Lee SK, Lee SC, Park JW, Kim SJ. The Utility of the Preoperative Neutrophilto-Lymphocyte Ratio in Predicting Severe Cholecystitis: A Retrospective Cohort Study. BMC Surg (2014) 14:100. doi: 10.1186/1471-2482-14-100

29. Suppiah A, Malde D, Arab T, Hamed M, Allgar V, Smith AM, et al. The Prognostic Value of the Neutrophil-Lymphocyte Ratio (NLR) in Acute Pancreatitis: Identification of an Optimal NLR. J Gastrointest Surg (2013) 17(4):675-81. doi: 10.1007/s11605-012-2121-1

30. Tanrikulu Y, Sen Tanrikulu C, Sabuncuoglu MZ, Kokturk F, Temi V, Bicakci E. Is the Neutrophil-to-Lymphocyte Ratio a Potential Diagnostic Marker for Peptic Ulcer Perforation? A Retrospective Cohort Study. Am J Emerg Med (2016) 34(3):403-6. doi: 10.1016/j.ajem.2015.11.009

31. De Larco JE, Wuertz BR, Furcht LT. The Potential Role of Neutrophils in Promoting the Metastatic Phenotype of Tumors Releasing Interleukin-8. Clin Cancer Res (2004) 10(15):4895-900. doi: 10.1158/1078-0432.ccr-03-0760

Conflict of Interest: The authors declare that the research was conducted in the absence of any commercial or financial relationships that could be construed as a potential conflict of interest.

Publisher's Note: All claims expressed in this article are solely those of the authors and do not necessarily represent those of their affiliated organizations, or those of the publisher, the editors and the reviewers. Any product that may be evaluated in this article, or claim that may be made by its manufacturer, is not guaranteed or endorsed by the publisher.

Copyright $\odot 2022 \mathrm{Gui}$, Liu, Shi, Xu, Qian and Wang. This is an open-access article distributed under the terms of the Creative Commons Attribution License (CC BY). The use, distribution or reproduction in other forums is permitted, provided the original author(s) and the copyright owner(s) are credited and that the original publication in this journal is cited, in accordance with accepted academic practice. No use, distribution or reproduction is permitted which does not comply with these terms. 